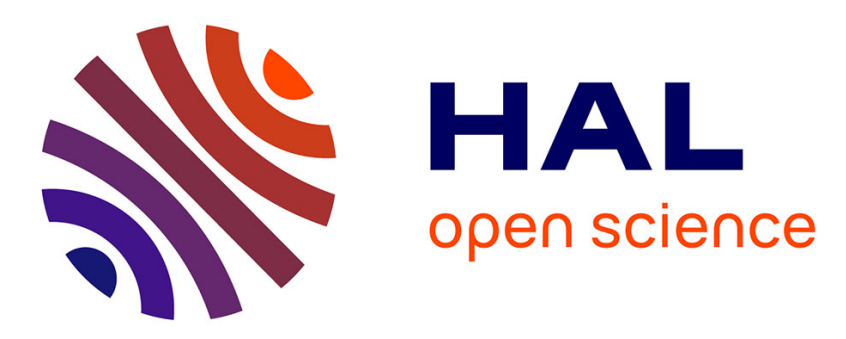

\title{
Electromagnetic Simulation of Power Modules via Adapted Modelling Tools
}

Enrico Vialardi, Edith Clavel, Olivier Chadebec, Jean-Michel Guichon, Marie

Lionet

\section{- To cite this version:}

Enrico Vialardi, Edith Clavel, Olivier Chadebec, Jean-Michel Guichon, Marie Lionet. Electromagnetic Simulation of Power Modules via Adapted Modelling Tools. EPE-PEMC 2010 14th International Power Electronics and Motion Control Conference, Sep 2010, Ohrid, Macedonia. hal-00521986

\section{HAL Id: hal-00521986 \\ https://hal.science/hal-00521986}

Submitted on 29 Sep 2010

HAL is a multi-disciplinary open access archive for the deposit and dissemination of scientific research documents, whether they are published or not. The documents may come from teaching and research institutions in France or abroad, or from public or private research centers.
L'archive ouverte pluridisciplinaire HAL, est destinée au dépôt et à la diffusion de documents scientifiques de niveau recherche, publiés ou non, émanant des établissements d'enseignement et de recherche français ou étrangers, des laboratoires publics ou privés. 


\title{
Electromagnetic Simulation of Power Modules via Adapted Modelling Tools
}

\author{
Enrico Vialardi*, Edith Clavel ${ }^{* *}$, Olivier Chadebec ${ }^{* *}$, Jean-Michel Guichon ${ }^{* *}$ and Marie Lionet ${ }^{*}$ \\ * CEDRAT, Technological Pole - Software, 38240 Meylan, France, e-mail: enrico.vialardi@ cedrat.com \\ ** Grenoble Electrical Engineering Laboratory - G2Elab, 38400 Saint Martin d'Hères, France
}

\begin{abstract}
Some modelling methods and associated tools are presented in this paper to efficiently and accurately simulate the electromagnetic behaviour of power electronic structures. Moreover, as engineers have to face new challenges, the synergy between simulation tools is illustrated as a possible solution for addressing this "multiphysic" topic. The article is also pictured of several application examples where obtained results are discussed and compared to measurements.
\end{abstract}

Keywords - Modelling, Simulation, Software, Power Converters.

\section{INTRODUCTION}

The efficient modelling and simulation of modern power modules is a great challenge, because their geometric and physical structure is becoming more and more complex and because the different electromagnetic phenomena to be take into account interrelate with each other.

Traditionally, modelling tools have been dedicated only to one specific issue which could be, for instance, the study of the performances of the cables or the analysis of a power converter or the design of an electrical motor. Nowadays this approach shows its limitations and it is no longer profitable for engineers that have to respect the time-to-market requirements during the design of a new product. In fact, the novel employed technologies, the strengthened standard limits to be respected and the growing performances desired by customers are transforming these distinct "uniphysic" problems into one "multiphysic" topic.

Consequently, the different modelling tools and the associated mathematical methods need to be strongly coupled with each other and to exchange data and results for ensuring a good representativeness of the simulated phenomena. Moreover, depending on the problem at hand it is advisable to use the best-adapted technique in order to reduce the CPU time and the memory requirements of the computation machines.

The aim of this paper is the presentation of the modelling process that can be followed to analyse some electromagnetic performances of a power converter, trying to take into account as many parameters as possible. Section II deals with three different modelling methods that are very valuable in the domain of power electronics: their main advantages and drawbacks are discussed and their respective application fields are listed. In Section III application examples are presented with a particular emphasis to the coupling between methods and to some industrial tools that have been applied for the simulation.
Finally, Section IV introduces a future integrated software environment where the complete "multiphysic" modelling of power modules could be performed.

\section{ADAPTED MODELLING METHODS}

As said above, the complete modelling of a power module integrated into its functioning environment requires specific and adapted tools. In other words, depending of the considered part of the system the mathematical methods and the associated models are not the same. In this section it is presented an overview of the three most valuable techniques: the Finite Element Method, the Partial Element Equivalent Circuit and the system-level simulation.

\section{A. The Finite Element Method}

The well-known Finite Element Method (FEM) is very useful and accurate to evaluate the electromagnetic fields of every complex complete structure. It is based on a volumic mesh of the domain and on the resolution of the Maxwell's equations at each node by means of pyramidshaped functions. Unfortunately, it requires the meshing of all the parts of the device (conductive, magnetic and dielectric elements) and also of the air region between them and of some free space surrounding the system.

Consequently, its use to achieve a complete modelling of a whole power converter is unrealistic because of the high number of unknowns needed for computing the behaviour of such a very large problem. On the other hand, the FEM method is unavoidable when magnetic materials are included in the structure: it is the case of the ferrite inductor constituting the EMC input filter of a power converter or of the mechanical box of a variable speed drive which is sometimes made of iron or steel. For these reasons, the industrial tool Flux ${ }^{\circledR}$ [1] which is based on the FEM and which has proven its efficiency for electrical design [2] has been used in this work for computing the magnetic field radiated by a three-phased inductor (see paragraph III.C below).

It is worth to note that the FEM results often expressed in the form of electromagnetic fields or potentials can be translated into equivalent electrical circuit in order to be exported towards system-level simulators like SPICE [3] or Portunus ${ }^{\circledR}$ [4].

\section{B. The PEEC Integral Method}

Despite the differential methods like FEM, integral techniques does not require to mesh all the free space around the device, but only active regions, like conductors, have to be taken into account. Therefore when the air volume is dominant, the integral methods are 
particularly attractive and their use becomes interesting and very efficient. However, at present time, the modelling of magnetic materials is still hard and not really profitable.

The Partial Element Equivalent Circuit (PEEC) technique is an integral method and is known in literature as one of the best-adapted approach for modelling every kind of metallic conductors, like for example the interconnection bars or the PCB traces between electric components [5]. In fact, it has the ability of attributing to each part (element) of the electromagnetic system an equivalent circuit made of a resistance and of inductances (self and mutual). In order to take into account the shape of the structure and the proximity and skin effects, a meshing of the conductors is however necessary, but for typical interconnection systems of power electronics the number of unknowns is very limited compared to the FEM.

In order to expand the frequency range of the $(\mathrm{R}, \mathrm{L})$ PEEC circuit, it is also possible to include parasitic capacitances computed by a dedicated integral method [6] or by the Adaptive Multi-Level Fast Multipole Method (AMLFMM).

In any case, the associated model is a large electrical equivalent circuit including resistances, inductances and mutual inductances which is frequency-dependent. The commercially available tool $\mathrm{InCa} \mathrm{D}^{\circledR}[7]$ is based on this method and was already widely used to model power electronics structures [8].

On one hand, solving the Kirchhoff's equations of the obtained circuit makes it possible to evaluate the current distribution inside structures like a power distribution system for instance. The near radiated field can be deduced by using Biot and Savart's law and then the electrodynamics effort by the Laplace's law. Or on the other hand, by reducing the electrical circuit, it is possible to obtain equivalent impedances of connections which can be then exported towards electrical simulators, such as SPICE or Portunus ${ }^{\circledR}$, for performing system level computations.

\section{The System-Level Simulation}

As presented above, most of the times, it is necessary to export the results of FEM or PEEC methods in order to achieve a system simulation, because a complete power electronics structure is made of passive (wires, PCB, ...) and active (diodes, transistors, IGBTs, ...) components. The first ones can be modeled using the PEEC or the FEM approaches, whereas the behaviour of active components needs to be modeled separately with specific non-linear techniques: some libraries are available according to the simulation tool used.

For power electronics applications, system-level simulation tools can be classified into two families: the SPICE-like environments and the mechatronic solvers. Several versions of SPICE-based simulators exist and are well-adapted when current and voltage waveforms are computed with standards components and when the complexity of the system is not very high.

Conversely, the interest for mechatronic tools lies in the different modelling approaches (block diagrams, electrical networks, state machines ...) that can be combined in the same software environment to perform accurate and integrated analog/mixed-signal simulations. As an example of system-level tool, Portunus ${ }^{\circledR}$ is applied in this work thanks to its continuous and time-adaptive solver which easily reaches the convergence. Well-provided libraries (electrical, mechanical, thermal, magnetic and power electronics) and the possibility for the user to define his own models by importing SPICE netlists, by Ccoding the behavioral equations or by defining components in the VHDL-AMS language represent a great benefit for performing any kind of transient or steady-state analysis. Moreover, the system-level simulation is a key point for engineers to comply with the "multiphysic" approach necessary during the design of a new power electronics product.

\section{SOME APPLICATION EXAMPLES}

In this paper, the previously-presented modelling methods are successfully applied to characterize industrial structures. Depending on the problem at hand, only one simulation technique is used or a coupling between methods is adopted. Sometimes this coupling is a simple modelling chain where output results of a technique become the input data of another tool. Elsewhere a strong synergy and a continuous data exchange between the methods are necessary to obtain accurate results.

This section is an overview of the possible computations that can be performed in power electronics with the above-detailed methods and tools. The advantages and drawbacks of each technique are also discussed and highlighted.

\section{A. Equivalent Impedances}

The first example deals with the evaluation of the parasitic behaviour of the interconnections (traces, ground planes, wire bonds, case ...) which are part of a power module. Their electromagnetic performances are typically estimated by means of equivalent impedances whose frequency dependence needs to be controlled by the designer.

The studied device shown in the top-left panel of Fig. 1 is a three-phased inverter $(600 \mathrm{~V}-75 \mathrm{~A})$ designed for $\mathrm{AC}$ motor control; its maximum switching frequency is 20 $\mathrm{kHz}$. It is provided of six control pins and multiple pins for power connections and it contains twelve semiconductor components (six IGBTs and six freewheeling diodes) to perform the desired switching functions (see the electrical circuit in Fig. 2).

The geometry of pins, wire bonds, DBC substrates as well as the heat spreader are described within $\mathrm{InCa} \mathrm{D}^{\circledR}$ in order to apply the PEEC method. Even if the heat spreader

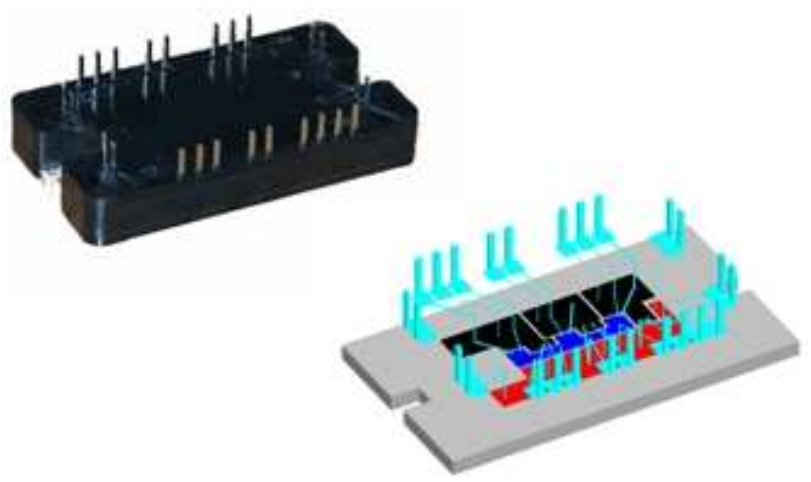

Fig. 1. The power module and its InCa3D representation 


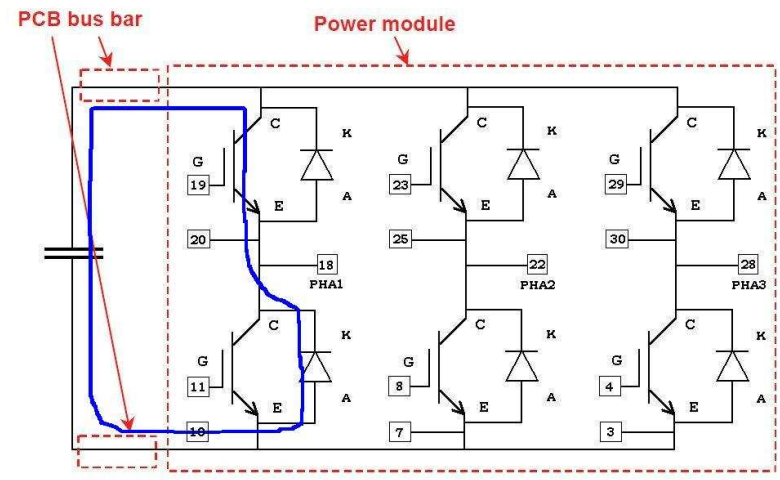

Fig. 2. Electrical circuit of the power module with one critical loop

does not have any electrical connection with the rest of the module, it acts as a ground plane and induces therefore an influence (via the mutual magnetic couplings) on the other conductors situated close to it. The InCa3 ${ }^{\circledR}$ description, reported in the bottom-right panel of Fig. 1, makes it possible to compute an electrical circuit constituted of many constant-value resistances and inductances (self and mutual) which are gathered into two matrices: $[\mathrm{R}]$ and $[\mathrm{L}]$.

The information contained in these matrices can be then compacted by means of a partial Gauss-Seidel procedure in order to compute the equivalent impedances seen from the device input/output ports defined by the designer. From the obtained model which is frequency-dependent, the evaluation of the parasitic impedance of the most critical electrical loops can be performed. As an example, the equivalent inductance of the loop indicated by the blue line in Fig. 2 is computed and compared to the measurements carried out on the real device. A very good agreement on the results can be observed: $75 \mathrm{nH}$ obtained by measurement and $71.5 \mathrm{nH}$ computed by $\mathrm{InCa}^{\circledR} \mathrm{D}^{\circledR}$ model.

The accurate estimation of the parasitic inductance of loops is a critical point in the power design since it is strongly linked to the overshoot amplitude of the drainsource voltage that occurs during switch commutation $(\mathrm{v}=\mathrm{L} \mathrm{di} / \mathrm{dt})$. As an example on the studied power converter a $V_{D S}$ overvoltage of $40 \mathrm{~V}$ has been measured.

\section{B. Current densities and Joule losses}

Since in the absence of magnetic materials the layout of a power electrical system can be efficiently modeled using the PEEC method, it is then natural to supply the obtained equivalent circuit with the generators and the loads. The solving of the associated Kirchhoff's equations leads to the evaluation of the current density inside each cell of the PEEC meshing. Moreover, the global current flowing in

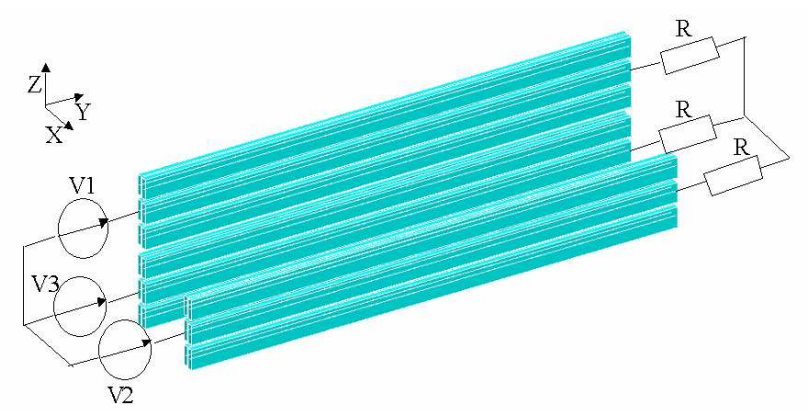

Fig. 3. The three-phased distribution system

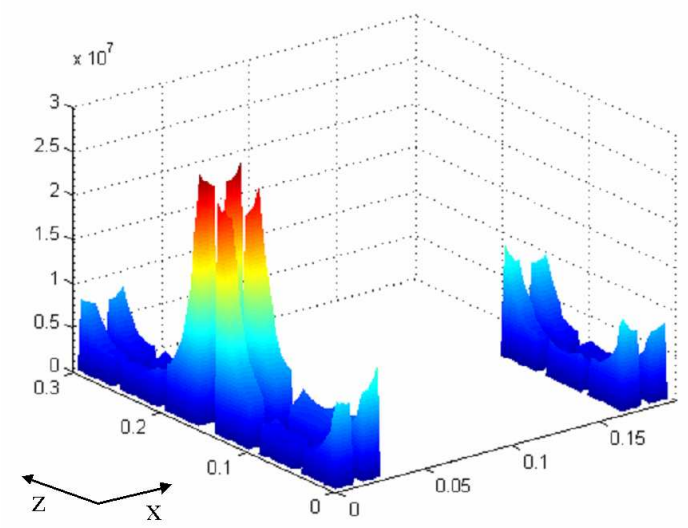

Fig. 4. Current density in the distribution system

the inputs/outputs of the structure can be computed as well as the Joule losses due to the resistive behaviour of the conductors.

This second example illustrates this topic and deals with a three-phased power distribution system composed of six conductors per phase, as depicted in Fig. 3. The presented modelling procedure is applied for the frequency of $50 \mathrm{~Hz}$ and the obtained spatial distribution of the current density is shown in Fig. 4, which emphasizes the skin and the proximity effects. In other words, the current density is not uniform in the section because of the shape and the geometric arrangement of the conductors. The concentration of the current near the external surface and near the other conductors means that the conductive material is badly used and additional Joule losses are generated.

It is possible to estimate the contribution of the two parasitic (skin and proximity) effects on the functional losses by means of two complementary simulations: the analysis of the system in DC conditions and the configuration where the three phases acts separately and does not couples with each other. The computed values summarized in Table I highlight that for the system at hand the proximity effect is dominant on the skin phenomenon and it provokes the Joule losses to quasidouble their impact.

TABLE I.

JOULE LOSSES ACCORDING TO SIMULATION CONDITIONS

\begin{tabular}{|c|c|c|}
\hline Simulation & Losses (W) & Ratio \\
\hline DC & 346 & 1 \\
\hline Skin effect only & 366 & 1.06 \\
\hline Skin and proximity effects & 613 & 1.77 \\
\hline
\end{tabular}

To improve the structure performances and the cabling effectiveness is consequently more advised to optimize the geometric arrangement of the bars rather than their section shape. A CFSQP (C code for Feasible Sequential Quadratic Programming) optimization algorithm [9] is applied to reduce the additional losses due to the position of the conductors under the constraint that the total space occupied by the distribution system does not vary. The original and the optimized arrangement are compared in Fig. 5: Joule losses are reduced of nearly $20 \%$, because the current density is more uniformly distributed in the section of conductors, as reported in Fig. 6. 


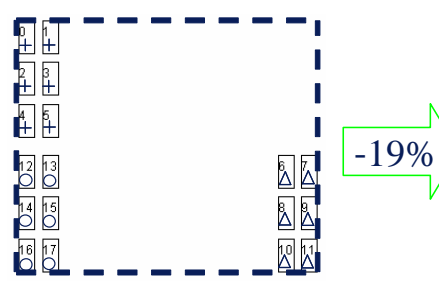

$613 \mathrm{~W}$

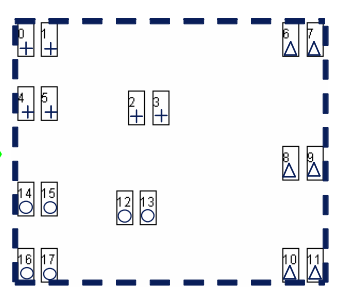

$487 \mathrm{~W}$
Fig. 5. Optimization process on the position of the bars

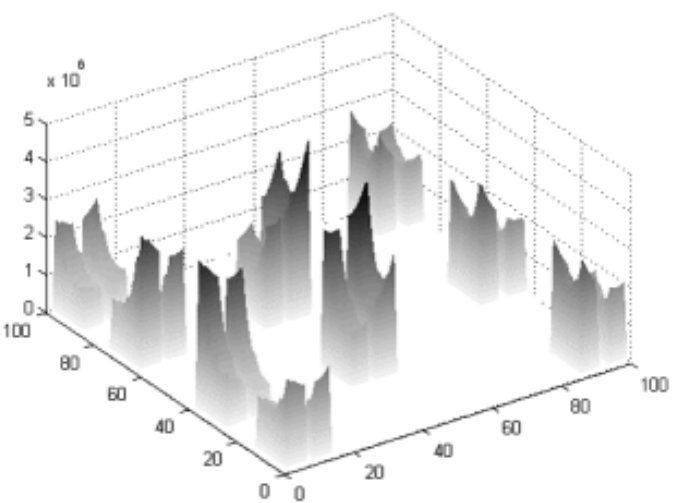

Fig. 6. Distribution of the current density after the optimization process

The analysis of the distribution of the current density and the Joule losses could also be considered as the starting point for the modelling of the thermal performances of the device. In fact, the regions where losses are highest represent the "hot points" of the structure and need to be studied via a coupled electrothermal tool. The procedure of this "multiphysic" approach, depicted in Fig. 7, is based on the realistic assumption that the temperature evolution does not affect the current repartition on the conductors, while it can modify the resistivity of the materials.

The Joule losses computed by the PEEC solver are exported to a thermal solver, able to take into account all modes of heat transfer (conduction, convection and radiation) and to provide the temperature distribution on the conductors. However, this temperature distribution usually differs from the reference-environment temperature (e.g., $20{ }^{\circ} \mathrm{C}$ ) at which the PEEC solver has evaluated the power losses. Consequently, an iterative process to readjust the data and the results is required.

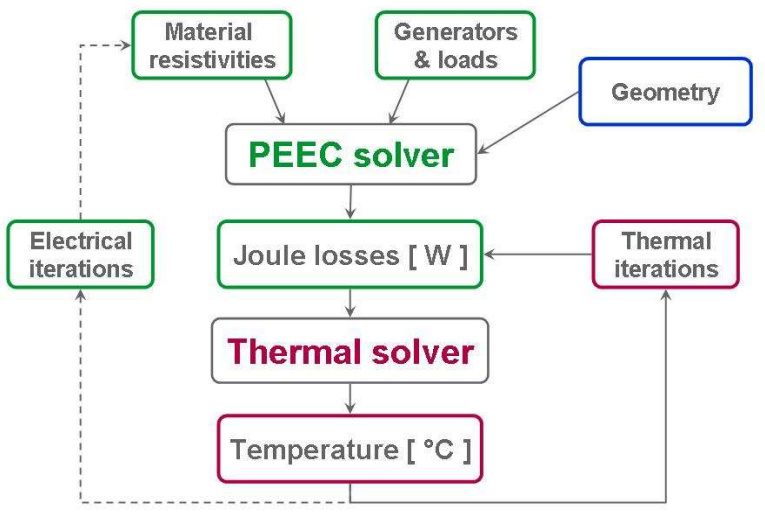

Fig. 7. Coupled electro-thermal simulation approach
In most of the practical cases, only internal iterations of the thermal solver are needed to reach the equilibrium results. Otherwise, especially when the temperature of the conductors is quite high, also the revision of the value of their resistivity and a new electrical PEEC computation are necessary. It is worth to note that even in worst cases one or two electrical iterations are enough for a good quality of the results.

\section{Magnetic Field}

Being known via the PEEC method the current density in each conductor, the Biot and Savart law, which is implemented in InCa3 ${ }^{\circledR}$, is able to compute the radiated magnetic field in the surrounding space as well as inside the conductors, with the assumption of a quasi-static approximation, i.e. in the near field zone.

This modelling approach is applied on the buck chopper converter shown in Fig. 8, which works at a switching frequency of $20 \mathrm{kHz}$. Its layout is especially designed to highlight the influence of three large current loops on the radiated magnetic field: three manual switches (S1, S2 and S3 in Fig. 8) alternatively activate these loops. In this study, the electronic components (e.g., semi-conductors, coils and capacitors) are simply modeled by equivalent RLC networks and Thévenin generators.

The magnetic field radiated at $20 \mathrm{kHz}$ at several points located $8 \mathrm{~cm}$ above the switching cell of the converter (see the red dashed line of Fig. 8) is computed by means of the PEEC method and the Biot and Savart law. Its amplitude, reported in Fig. 9, agrees quite well with the real measurements: the differences can be ascribed, among others, to the ferromagnetic-core coils, which are not

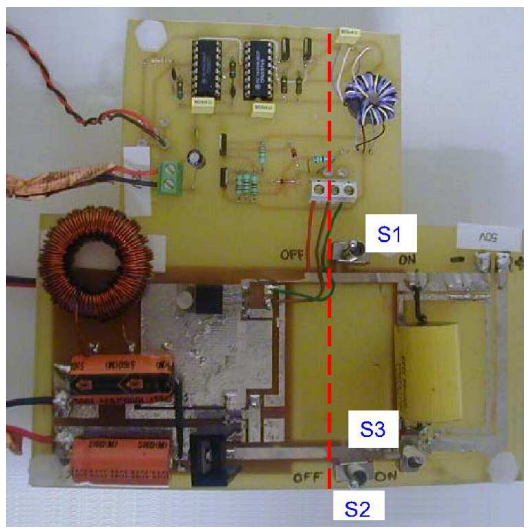

Fig. 8. Layout of the buck chopper converter

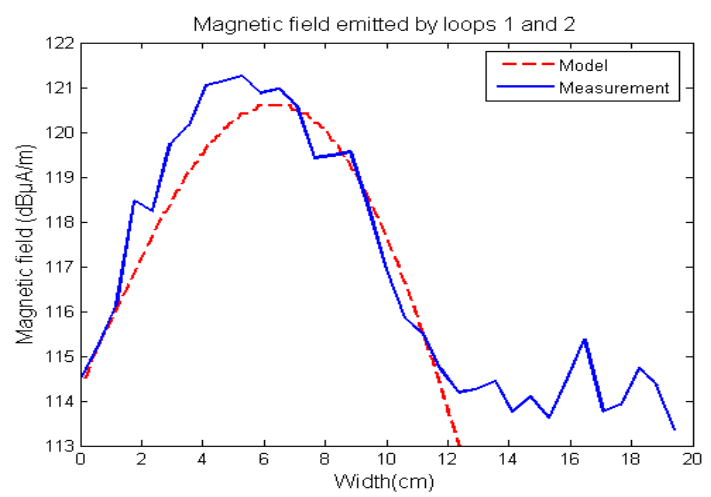

Fig. 9. Amplitude of the magnetic field radiated at the frequency of $20 \mathrm{kHz}$ at $8 \mathrm{~cm}$ above the converter 


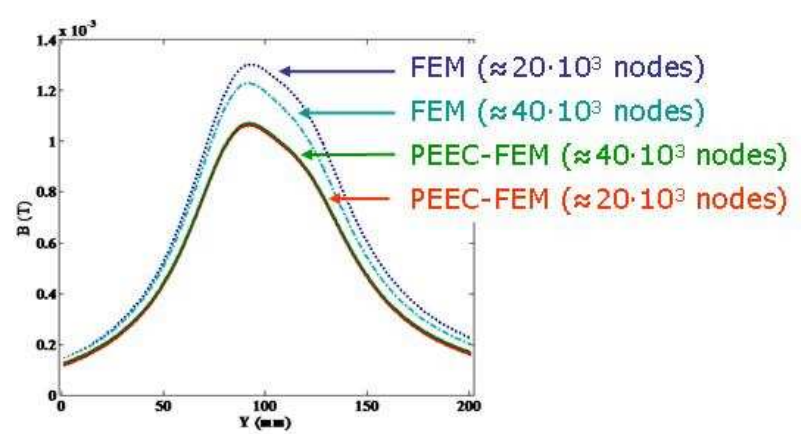

Fig. 10. Validation of the coupled PEEC-FEM approach

modeled and meshed as 3D elements. In fact, the PEEC methodology is poorly adapted in terms of CPU time and memory requirements when the considered structure includes magnetic materials. Some theoretical extensions of PEEC have been proposed [11] but their validity domain is limited to simple geometries.

On the other hand, the FEM method is still the bestadapted tool for modelling magnetic materials and its coupled use with the PEEC approach is the winning strategy for analyzing structures composed of conductors and magnetic parts. The coupling between these two methods has been developed [12] and it is applied in this work to model a power converter, which disposes of an EMC input filter composed of a toroidal core of ferrite and three winded wires.

Preliminary results of the global magnetic field density radiated by the whole structure at a distance of $50 \mathrm{~mm}$ are shown in Fig. 10, where the higher efficiency of the PEEC-FEM approach is emphasized in comparison with the full FEM model. In fact, about 20 thousands nodes are enough for the coupled methodology to obtain excellent results, whereas the Finite Elements analysis does not achieve an accurate computation, even with 40 thousands mesh elements.

\section{Electrodynamic Stress}

The estimation of the current density and then of the magnetic field density makes it possible to compute, by means of the Laplace law, the electrodynamic efforts acting on conductors. As their amplitude becomes particularly critical in case of short-circuit and can provoke the mechanical deformation of conductors, it is advised to introduce these results into a mechanical tool to evaluate the strength of materials and eventually their deformation or failure.

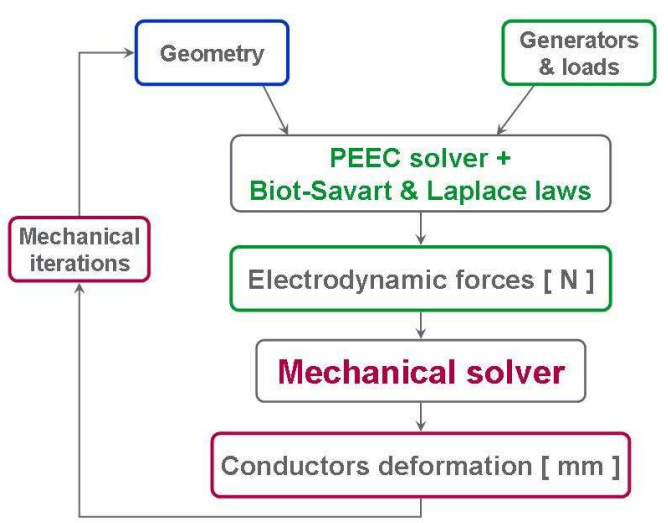

Fig. 11. Coupled electro-mechanical simulation approach

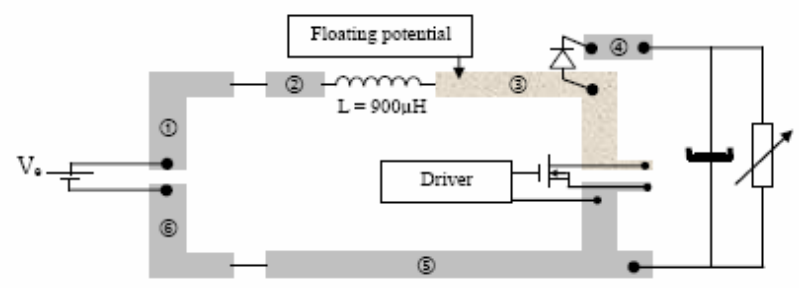

Fig. 12. Boost converter topology

As the geometry of the structure (i.e., the shape and the arrangement of conductors) is a sensitive parameter for the flowing currents and for the electrodynamic stresses, an iterative process between the electrical and the mechanical simulations has to be set-up for obtaining accurate results. Such a procedure is illustrated in Fig. 11.

\section{E. Electric Circuit and Time-Domain Waveforms}

The last example deals with the integration in a systemlevel simulator of some equivalent models computed on specific parts of the system by means of dedicated 3D tools. In particular, the time-domain performances of a boost converter (see Fig. 12) are finally analyzed by Portunus $^{\circledR}$ and compared to measurements.

The low-frequency (resistive and inductive) behaviour of the interconnections (PCB traces and ground plane) is modeled with InCa3D ${ }^{\circledR}$ and exported towards Portunus ${ }^{\circledR}$ in the form of an equivalent impedance matrix. Meanwhile, the equivalent capacitances representing the electric couplings between the conductors are computed by means of the AMLFMM algorithm [6], which is also able to take into account the influence of the PCB dielectric substrate.

The R-L matrix and these self and mutual capacitances are elements of a global electric circuit constituted also by the appropriated models of active components (battery and drive circuit) and semiconductors (diodes and IGBT). The simulation of the whole system must be a transient analysis, because some models are non-linear and consequently the principle of the Fourier transform does not yet apply.

The performed Portunus ${ }^{\circledR}$ computations are focused on the IGBT commutations, since these events are the most critical ones from an EMC point of view: fast switchings are responsible of electromagnetic noise that can perturb the system behaviour. In this example the drain-source voltage of the IGBT and the common mode current flowing in the mechanical ground are considered.
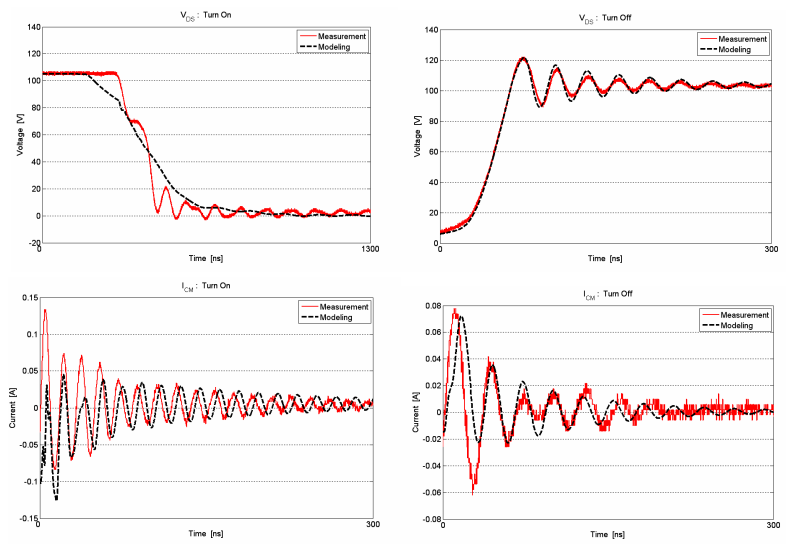

Fig. 13. Drain-source voltages (top panels) and common mode currents (bottom panels) at turn-on and turn-off commutations 
The obtained waveforms are reported in Fig. 13, where they are successfully compared with the measurements performed on a real mock-up of the converter [13]. It is worth to note that the waveform oscillations are correctly modeled thanks to the inclusion of the parasitic capacitances into the equivalent circuit.

\section{TOWARDS AN INTEGRATED ENVIRONMENT}

The previous section has listed some analysis that can be achieved using the modelling methods presented in section II. The evaluated data are very helpful for engineers in order to make the design more efficient, but an integrated environment will further reduce time and costs of the development process. The fulcrum of such a platform is the system-level simulator, which drives the different 3D tools and manages the data exchanges thanks to the API (Application Programming Interface) technology.

Some links are already active and have been applied in this work: the import of InCa3D ${ }^{\circledR}$ equivalent macro-blocks inside Portunus ${ }^{\circledR}$ or the coupling between FEM and PEEC methods to model magnetic conductors, for example. The feasibility and the interest of other dialogues have been demonstrated and need to be implemented in order to reach the final goal of a "multiphysic" platform for power electronics.

\section{REFERENCES}

[1] Flux ${ }^{\circledR}$ software, Cedrat, http://www.cedrat.com

[2] D. G. Dorrell, "Design Requirements for Brushless Permanent Magnet Generators for Use in Small Renewable Energy Systems",
IECON2007, Taipei, pp.216-221, November 2007.

[3] L. W. Nagel, D. O. Pederson, "SPICE (Simulation Program with Integrated Circuit Emphasis)", Memorandum ERL-M382, University of California, Berkeley, April 1973.

[4] Portunus $^{\circledR}$ software, Adapted Solutions, http://www.adaptedsolutions.com

[5] J-P. Gonnet, E. Clavel, V. Mazauric, J. Roudet, "PEEC Method Dedicated to the Design of Electrical Systems", PIERSO4, Pise, Italy, pp. 493-496, March 2004.

[6] V. Ardon, J. Aimé, O. Chadebec, E. Clavel, E. Vialardi, "MoM and PEEC Method to Reach a Complete Equivalent Circuit of a Static Converter", EMC Zurich 2009, Zurich, Switzerland, pp. 273-276, January 2009.

[7] InCa3 $\mathrm{D}^{\circledR}$ software, Cedrat, http://www.cedrat.com

[8] R. Prades, M. Lionet, X. Brunotte, Y. Le Floch, E. Clavel, J-L. Schanen, J-M. Guichon, "Improving Conducted EMI Forecasting with Accurate Layout Modeling", 2EMC 2007, Rouen, France, session 7A, October 2007.

[9] C. T. Lawrence, "A Computationally Efficient Feasible Sequential Quadratic Programming Algorithm", Ph.D. thesis, University of Maryland, College Park, MD, 1998.

[10] J. Aimé, J. Roudet, E. Clavel, O. Aouine, C. Labarre, F. Costa, J. Ecrabey, "Prediction and measurement of the magnetic near field of a static converter", IEEE-ISIE 2007, Vigo, Spain, pp. 25502555, June 2007.

[11] J-P. Gonnet, E. Clavel, V. Mazauric, J-M. Guichon, J. Roudet, "Method for an optimal design of interconnections busbars", IEEE-CEFC'04, Seoul, Korea, pp. 26, June 2004.

[12] T. S. Tran, G. Meunier, P. Labie, Y Le Floch, J. Roudet, J-M. Guichon, Y. Maréchal, "Coupling PEEC-Finite Element Method for Solving Electromagnetic Problem", COMPUMAG 2007, Aachen, Germany, June 2007.

[13] J. Aimé, J. Roudet, C. Vollaire, P. Baudesson, J. Ecrabey, "Layout techniques for reduction of common mode current in static converters", 2006 IEEE-IAS Annual Meeting Report, vol. 5, pp. 2296-2303, October 2006. 